International Journal of Pure and Applied Mathematics

Volume 87 No. $6 \quad 2013,781-793$

ISSN: 1311-8080 (printed version); ISSN: 1314-3395 (on-line version)

url: http://www.ijpam.eu

doi: http://dx.doi.org/10.12732/ijpam.v87i6.7

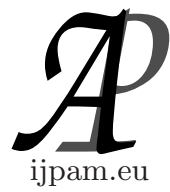

\title{
ON VERTEX DISJOINT CYCLES OF MIDDLE AND TOTAL GRAPH OF $K_{n}$ AND $K_{m, n}$
}

\author{
M.M. Akbar Ali \\ Department of Mathematics \\ Karunya University \\ Coimbatore, 641 114, Tamil Nadu, INDIA
}

\begin{abstract}
Tulgeity $\tau(G)$ of a graph $G$ is the maximum number of vertex disjoint cycles contained in $G$. In this paper the basic results on tulgeity of a graph have been reviewed and the formula for the tulgeity of the middle and total graph of complete graph and complete bigraph are derived. Also an upper bound for the tulgeity of middle graph of any graph is presented and the graph for which the tulgeity attains its upper bound has been classified.
\end{abstract}

AMS Subject Classification: 05C70, 05C75, 05C76

Key Words: tulgeity, line graph, total graph, complete graph and complete bigraph

\section{Introduction}

Cycle is a significant feature of a graph. The analysis of cycle plays an important role in the design and development of some graph model. Several problems of finding shortest path, shortest spanning trees, least cost Hamiltonian cycles, etc., of a graph have been studied. We discuss the problems of finding the maximum number of vertex disjoint cycles.

We consider finite, simple, undirected graph $G(V(G), E(G))$ where $V(G)$ and $E(G)$ represent vertex set and edge set of $G$ respectively. $p$ and $q$ denote the

Received: September 6, 2013

(c) 2013 Academic Publications, Ltd. url: www.acadpubl.eu 
number of vertices and edges of the graph $G$ respectively. For any real number $r,[r]$ and $\lceil r\rceil$ denote the largest integer not exceeding $r$ and the least integer not less than $r$, respectively. If $\mathbb{C}$ is the collection of cycles of a graph then $V(\mathbb{C})$ denotes the set of vertices belonging to the cycles of $\mathbb{C}$. $\langle S\rangle$ denotes the subgraph of $G$ induced by the set of vertices $S \subseteq V(G)$. The other notations and terminology used in this paper can be found in [5].

Graph can be characterized by a type of configuration or subgraph they possess. In [3], Gary Chartrand et al., characterized several classes of graphs by defining them with the property $P_{n}$. A graph $G$ has the property $P_{n}$, where $n$ is a positive integer, if $G$ contains no subgraph which is homeomorphic from the complete graph $K_{n+1}$ or the complete bigraph $K\left(\left[\frac{n+2}{2}\right]\left\lceil\frac{n+2}{2}\right\rceil\right)$. Trivially, a graph $G$ has the property $P_{2}$, if $G$ contains no cycles. They introduced the concept of point partition number and dual point partition number of a graph $G$. The point partition number of a graph is defined as the minimum number of subsets into which the vertex set of $G$ can be partitioned so that the subgraph induced by each subset has the property $P_{n}$. Dual to this concept the dual point partition number of a graph is defined as the maximum number of subsets into which the vertex set of $G$ can be Partitioned so that the subgraph induced by each subset does not possesses the property $P_{n}$. The problems of Nordhans - Gaddum type for the dual point partition number are investigated in $[6]$.

In [4] Gary Chartrand et al., defined the term tulgeity of a graph as the dual point partition number for $n=2$.(i.e) Tulgeity is the maximum number of subsets into which the vertex set of $G$ can be partitioned so that the subgraph induced by each subset contains a cycle. Equivalently the tulgeity of a graph is the maximum number of vertex disjoint cycles in $G$ and it is denoted by $\tau(G)$. Since every cycle contains at least three vertices, an obvious upper bound for tulgeity of any graph $G$ with $p$ vertices is $\tau(G) \leq[p / 3]$ and $\tau(G)=[p / 3]$ if $G$ is a complete graph with $p$ vertex.

Middle graph $M(G)$ of a graph $G$ is defined with the vertex set $V(G) \cup E(G)$, in which two elements are adjacent if and only if either both are adjacent edges in $G$ or one of the elements is a vertex and the other one is an edge incident to the vertex in $G$.

Total graph $T(G)$ of a graph $G$ defined with the vertex set $V(G) \cup E(G)$, in which two elements are adjacent if and only if one of the following holds true (i) both are adjacent edges or vertices in $G$ (ii) one is a vertex and other is an edge incident to it in $G$. 


\section{Basic Results}

We begin by presenting the existing results concerning the tulgeity of a graph.

Theorem 2.1. [4] For any graph $G, \tau(G)=\sum \tau(C) \leq \sum \tau(B)$, where the sums being taken over all components $C$ and blocks $B$ of $G$, respectively.

Theorem 2.2. [4] For the complete n-partite graph $G=K\left(p_{1}, p_{2}, \ldots, p_{n}\right), 1 \leq$ $p_{1} \leq p_{2} \leq \ldots . \leq p_{n}$ and $\sum p_{i}=p, \tau(G)=\min \left(\left[\frac{1}{2} \sum_{0}^{n-1} p_{i}\right],[p / 3]\right)$, where $p_{0}=0$.

We have derived the formula to find the tulgeity of the line graph of complete graph and complete bigraph.

Theorem 2.3. $[1] \tau\left(L\left(K_{n}\right)\right)=\left[\frac{n(n-1)}{6}\right]$

Theorem 2.4. $[1] \tau\left(L\left(K_{m, n}\right)=\left[\frac{m n}{3}\right]\right.$

Also, we have derived an upper bound for the tulgeity of line graph of any graph and characterized the graphs for which the tulgeity attains its upper bound.

Theorem 2.5. [1] For any graph $G, \tau(L(G)) \leq \sum_{i}\left[\frac{\operatorname{deg} v_{i}}{3}\right]$ where $\operatorname{deg} v_{i}$ denotes the degree of the vertex $v_{i}$ and the the summation taken over all the vertices of $G$.

Theorem 2.6. [1] If $G$ is a tree and for each pair of vertices $\left(v_{i}, v_{j}\right)$ with $\operatorname{deg} v_{i}, \operatorname{deg} v_{j}>2$, if there exist a vertex $v$ of degree 2 on $P\left(v_{i}, v_{j}\right)$ then $\tau(L(G)) \leq \sum_{i}\left[\frac{\operatorname{deg} v_{i}}{3}\right]$.

Also we have derived [2] the results to find the tulgeity of line, middle and total graph of Wheel graph, Gear graph and Helm graph.

\section{Tulgeity of Middle graph of $K_{n}$}

Theorem 3.1. The tulgeity of middle graph of $K_{n}(n \geq 4), \tau\left(M\left(K_{n}\right)\right)=$ $\left[\frac{n(n+1)}{6}\right]$

Proof. Let $V\left(K_{n}\right)=\left\{v_{i}: 1 \leq i \leq n\right\}$ and $E\left(K_{n}\right)=\left\{e_{i j}=v_{i} v_{j}: 1 \leq\right.$ 
$i \leq n, i+1 \leq j \leq n\}$. By the definition of middle graph, $V\left(M\left(K_{n}\right)\right)=$ $V\left(K_{n}\right) \cup E\left(K_{n}\right)$.

If $n=4$, then $V\left(M\left(K_{4}\right)\right)=\left\{v_{i}: 1 \leq i \leq 4\right\} \cup\left\{e_{12}, e_{13}, e_{14}, e_{23}, e_{24}, e_{34}\right\}$ and there exists the collection $\mathbb{C}=\left\{e_{12} v_{2} e_{23}, e_{13} v_{3} e_{34}, e_{14} e_{24} v_{4}\right\}$ of disjoint cycles of $M\left(K_{4}\right)$. Hence $\tau\left(M\left(K_{4}\right)\right)=\left[\frac{n(n+1)}{6}\right]$ where $n=4$.

If $n=5$, then $V\left(M\left(K_{n}\right)\right)=\left\{v_{i}: 1 \leq i \leq 5\right\} \cup\left\{e_{12}, e_{13}, e_{14}, e_{15}, e_{23}, e_{24}\right.$, $\left.e_{25}, e_{34}, e_{35}, e_{45}\right\}$ and there exists the collection $\mathbb{C}=\left\{v_{1} e_{12} e_{15}, v_{2} v_{23} e_{25}, v_{3} e_{13}\right.$ $\left.e_{34}, v_{4} e_{14} e_{24}, v_{5} e_{35} e_{45}\right\}$ of disjoint cycles of $M\left(K_{5}\right)$. Hence

$$
\tau\left(M\left(K_{5}\right)\right)=\left[\frac{n(n+1)}{6}\right],
$$

where $n=5$.

Assume $n>5$. For each $i(1 \leq i \leq n),<\left\{v_{i}\right\} \cup\left\{e_{i j}: i+1 \leq j \leq n\right\}>$ is a clique (say $K_{i}^{\prime}$ ) of order $n-i+1$ in $M\left(K_{n}\right)$.

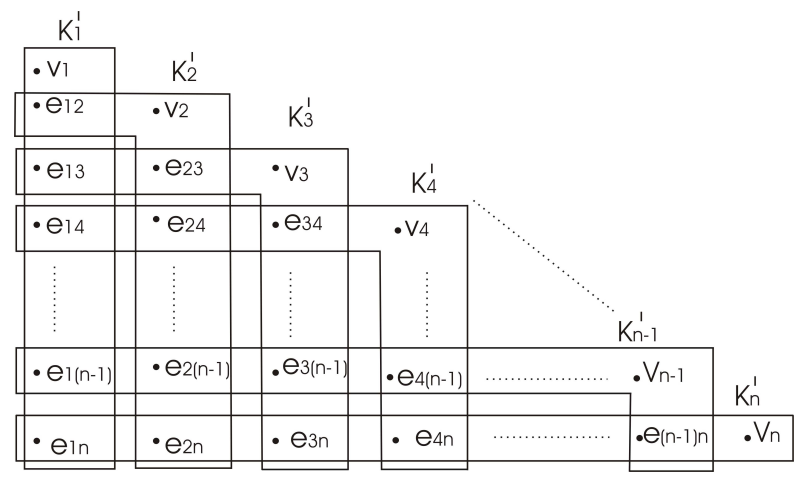

Cliques in $M\left(K_{n}\right)$

Figure 3.1

If $V\left(K_{l}^{\prime}\right) \cap V\left(K_{m}^{\prime}\right) \neq \phi$ for any $l \neq m$ then there exists a vertex $e_{i k} \in V\left(K_{l}^{\prime}\right) \cap$ $V\left(K_{m}^{\prime}\right)$. (ie) $e_{i k} \in\left\{v_{l}\right\} \cup\left\{e_{l j}: l+1 \leq j \leq n\right\}$ and $e_{i k} \in\left\{v_{m}\right\} \cup\left\{e_{m j}: m+1 \leq j \leq\right.$ $n\}$. (ie) $e_{i k} \in\left\{e_{l j}: l+1 \leq j \leq n\right\}$ and $e_{i k} \in\left\{e_{m j}: m+1 \leq j \leq n\right\}$. (ie) $i=l$ and $i=m \Rightarrow l=m$. This contradiction shows that $V\left(K_{l}^{\prime}\right) \cap V\left(K_{m}^{\prime}\right)=\phi$ for $l \neq m$. Also $\bigcup_{i=1}^{n} V\left(K_{i}^{\prime}\right)=V\left(M\left(K_{n}\right)\right)$. Hence $\tau\left(M\left(K_{n}\right)\right) \geq \sum_{i=1}^{n} \tau\left(K_{i}^{\prime}\right)$. Clearly for each $i(1 \leq i \leq n),\left|V\left(K_{i}^{\prime}\right)\right|=n-i+1$.

case $(\mathbf{i})(n \equiv 0(\bmod 3))$

For each $i=3 k-2,\left(1 \leq k \leq \frac{n}{3}\right),\left|V\left(K_{i}^{\prime}\right)\right|=n+3-3 k \equiv 0(\bmod 3)$. Hence there exists $\frac{1}{3}\left|V\left(K_{i}^{\prime}\right)\right|$ vertex disjoint 3-cycles in $K_{i}^{\prime}$ for each $i=3 k-2(1 \leq k \leq$ 
$\left.\frac{n}{3}\right)$. We find the partition of $V\left(M\left(K_{n}\right)\right)$ as follows. $V\left(M\left(K_{n}\right)\right)=V^{\prime} \cup V^{\prime \prime}$ where $V^{\prime}=\bigcup_{i=1}^{n-6} V\left(K_{i}^{\prime}\right)$ and $V^{\prime \prime}=\bigcup_{i=n-5}^{n} V\left(K_{i}^{\prime}\right)$. It is clear that $\tau\left(M\left(K_{n}\right)\right) \geq \tau\left(<V^{\prime}>\right.$ )$+\tau\left(<V^{\prime \prime}>\right)$. $V^{\prime}$ can be expressed as

$$
V^{\prime}=\bigcup_{k=1}^{\frac{n}{3}-2} V\left(K_{3 k-2}^{\prime}\right) \bigcup_{k=1}^{\frac{n}{3}-2} V\left(K_{3 k-1}^{\prime}\right) \bigcup_{k=1}^{\frac{n}{3}-2} V\left(K_{3 k}^{\prime}\right)
$$

(ie) $\tau\left(<V^{\prime}>\right)=\sum_{k=1}^{\frac{n}{3}-2} \tau\left(K_{3 k-2}^{\prime}\right)+\sum_{k=1}^{\frac{n}{3}-2} \tau\left(K_{3 k-1}^{\prime}\right)+\sum_{k=1}^{\frac{n}{3}-2} \tau\left(K_{3 k}^{\prime}\right)$. For each $k \quad(1 \leq$ $\left.k \leq \frac{n}{3}-2\right),\left|V\left(K_{3 k-2}^{\prime}\right)\right|=n-3 k+3 \equiv 0(\bmod 3)$ and hence $K_{3 k-2}^{\prime}$ is a clique with $\frac{1}{3}\left|V\left(K_{3 k-2}^{\prime}\right)\right|$ vertex disjoint 3 -cycles. Hence $\tau\left(K_{3 k-2}^{\prime}\right)=\frac{1}{3}(n-3 k+3)=$ $\frac{n}{3}-k+1$ for each $k\left(1 \leq k \leq \frac{n}{3}-2\right)$. $\sum_{k=1}^{\frac{n}{3}-2} \tau\left(K_{3 k-2}^{\prime}\right)=\sum_{k=1}^{\frac{n}{3}-2}\left(\frac{n}{3}-k+1\right)=$ $\frac{1}{2}\left(\frac{n}{3}-2\right)\left(\frac{n}{3}+3\right)$. For each $k\left(1 \leq k \leq \frac{n}{3}-2\right)\left|V\left(K_{3 k-1}^{\prime}\right)\right|=n-3 k+$ $2 \equiv 2(\bmod 3)$ and $\left|V\left(K_{3 k}^{\prime}\right)\right|=n-3 k+1 \equiv 1(\bmod 3)$. Let $K_{3 k-1}^{\prime \prime}=$ $K_{3 k-1}^{\prime}-\left\{e_{(3 k-1) 3 k}, e_{(3 k-1)(3 k+1)}\right\}$ and $K_{3 k}^{\prime \prime}=K_{3 k}^{\prime}-\left\{e_{(3 k)(3 k+1)}\right\}$ for each $k$, $\left(1 \leq k \leq \frac{n}{3}-2\right)$. It is clear that $\left|V\left(K_{3 k-1}^{\prime \prime}\right)\right|=\left|V\left(K_{3 k}^{\prime \prime}\right)\right|=n-3 k \equiv 0(\bmod 3)$ for each $k\left(1 \leq k \leq \frac{n}{3}-2\right)$. Also $<\left\{e_{(3 k-1) 3 k}, e_{(3 k-1)(3 k+1)}, e_{3 k(3 k+1)}\right\}>$ is a 3 cycle for each $k\left(1 \leq k \leq \frac{n}{3}-2\right)$. Hence $\sum_{k=1}^{\frac{n}{2}-3} \tau\left(K_{3 k-1}^{\prime}\right)+\sum_{k=1}^{\frac{n}{2}-3} \tau\left(K_{3 k}^{\prime}\right)=$ $\sum_{k=1}^{\frac{n}{2}-3}\left(\frac{1}{3}\left|V\left(K_{3 k-1}^{\prime \prime}\right)\right|+\frac{1}{3}\left|V\left(K_{3 k}^{\prime \prime}\right)\right|+1\right)=\sum_{k=1}^{\frac{n}{2}-3}\left(\frac{1}{3}(n-3 k)+\frac{1}{3}(n-3 k)+1\right)=$ $\left(\frac{n}{3}-2\right)\left(\frac{n}{3}+2\right)$. Since $V^{\prime \prime}=\bigcup_{i=n-5}^{5} V\left(K_{i}^{\prime}\right),\left|V^{\prime \prime}\right|=6+5+\cdots+1=21 \equiv$ $0(\bmod 3)$. We find the following collection of seven disjoint 3 -cycles of $<V^{\prime \prime}>$

$$
\begin{array}{r}
\mathbb{C}_{i}=\left\{v_{n-5} e_{(n-5)(n-4)} e_{(n-5)(n-3)}, e_{(n-5)(n-2)} v_{n-2} e_{(n-2)(n-1)}, e_{(n-5)(n-1)}\right. \\
v_{n-1} e_{(n-4)(n-1)}, e_{(n-5) n} e_{(n-4) n} e_{(n-3) n}, e_{(n-4)(n-3)} v_{n-4} e_{(n-4)(n-2)}, e_{(n-3)(n-2)} \\
\left.v_{n-3} e_{(n-3)(n-1)}, e_{(n-2) n} v_{n} e_{(n-1) n}\right\} .
\end{array}
$$

Hence $\tau\left(<V^{\prime \prime}>\right)=7$.

$$
\begin{aligned}
\tau\left(M\left(K_{n}\right)\right) \geq \tau & \left(<V^{\prime}>\right)+\tau\left(<V^{\prime \prime}>\right) \\
& =\frac{\left(\frac{n}{3}-2\right)\left(\frac{n}{3}+3\right)}{2}+\left(\frac{n}{3}-2\right)\left(\frac{n}{3}+2\right)+7
\end{aligned}
$$




$$
=\frac{n(n+1)}{6}
$$

case (ii) $(n \equiv 1(\bmod 3))$

Set $V^{\prime}=V\left(K_{1}^{\prime}\right) \bigcup_{i=2}^{n-6} V\left(K_{i}^{\prime}\right), V^{\prime \prime}=\bigcup_{i=n-5}^{n} V\left(K_{i}^{\prime}\right)$ so that $V\left(M\left(K^{\prime}\right)\right)=V^{\prime} \cup$ $V^{\prime \prime}$.

$$
V^{\prime}=V\left(K_{1}^{\prime}\right) \bigcup_{i=2}^{n-6} V\left(K_{i}^{\prime}\right)=V\left(K_{1}^{\prime}\right) \bigcup_{k=1}^{\frac{n-7}{3}} V\left(K_{3 k-1}^{\prime}\right) \bigcup_{k=1}^{\frac{n-7}{3}} V\left(K_{3 k}^{\prime}\right) \bigcup_{k=1}^{\frac{n-7}{3}} V\left(K_{3 k+1}^{\prime}\right) .
$$

Since $\left|V\left(K_{1}^{\prime}\right)\right|=n \equiv 1(\bmod 3), K_{1}^{\prime}$ has $\frac{n-1}{3}$ vertex disjoint 3 -cycles. For each $k\left(1 \leq k \leq \frac{n-7}{3}\right),\left|V\left(K_{3 k-1}^{\prime}\right)\right|=n-3 k+2 \equiv 0(\bmod 3)$ and hence $\tau\left(K_{3 k-1}^{\prime}\right)=\frac{1}{3}(n-3 k+2),\left(1 \leq k \leq \frac{n-7}{3}\right)$. Hence

$$
\sum_{k=1}^{\frac{n-7}{3}} \tau\left(K_{3 k-1}^{\prime}\right)=\frac{1}{3} \sum_{k=1}^{\frac{n-7}{3}}(n-3 k+2)=\frac{(n-7)(n+8)}{18} .
$$

For each $k\left(1 \leq k \leq \frac{n-7}{3}\right)$,

$$
\left|V\left(K_{3 k}^{\prime}\right)\right|=n-3 k+1 \equiv 2(\bmod 3)
$$

and

$$
\left|V\left(K_{3 k+1}^{\prime}\right)\right|=n-3 k-1+1=n-3 k \equiv 1(\bmod 3) .
$$

Let $K_{3 k}^{\prime \prime}=K_{3 k}^{\prime}-\left\{e_{(3 k)(3 k+1)}, e_{(3 k)(3 k+2)}\right\}$ and $K_{3 k+1}^{\prime \prime}=K_{3 k+1}^{\prime}-\left\{e_{(3 k+1)(3 k+2)}\right\}$ for each $k\left(1 \leq k \leq \frac{n-7}{2}\right)$. It is clear that $\left|V\left(K_{3 k}^{\prime \prime}\right)\right|=n-3 k-1 \equiv 0(\bmod 3)$ and $\left|V\left(K_{3 k+1}^{\prime \prime}\right)\right|=n-3 k-1 \equiv 0(\bmod 3)$ for each $k\left(1 \leq k \leq \frac{n-7}{3}\right)$. Also for each $k\left(1 \leq k \leq \frac{n-7}{3}\right),<\left\{e_{(3 k)(3 k+1)}, e_{(3 k)(3 k+2)}, e_{(3 k+1)(3 k+2)}\right\}>$ is a $3-$ cycle. Hence $\sum_{k=1}^{\frac{n-7}{3}} \tau\left(K_{3 k}^{\prime}\right)+\sum_{k=1}^{\frac{n-7}{3}} \tau\left(K_{3 k+1}^{\prime}\right)=\frac{(n-7)(n+8)}{18}$. Since $\left|V^{\prime \prime}\right|=$ $\sum_{i=n-5}^{n}\left|V\left(K_{i}^{\prime}\right)\right|=\sum_{i=n-5}^{n}(n-i+1)=21 \equiv 0(\bmod 3)$. There exists the following collection $\mathbb{C}$ of vertex disjoint cycles in $\left\langle V^{\prime \prime}\right\rangle$

$$
\begin{gathered}
\mathbb{C}=\left\{v_{n-5} e_{(n-5)(n-3)} e_{(n-5)(n-2)}, e_{(n-5)(n-4)} v_{n-4} e_{(n-4)(n-2)},\right. \\
e_{(n-5)(n-1)} e_{(n-4)(n-1)} e_{(n-3)(n-1)}, e_{(n-5) n} e_{(n-4) n} e_{(n-1) n}, e_{(n-4)(n-3)} v_{n-3} e_{(n-3) n}, \\
\left.e_{(n-3)(n-2)} v_{n-2} e_{(n-2) n}, e_{(n-2)(n-1)} v_{n-1} e_{(n-1) n}\right\} .
\end{gathered}
$$


Hence $<V^{\prime \prime}>=7$. Therefore

$$
\begin{aligned}
\tau\left(M\left(K_{n}\right)\right) & \geq \tau\left(K_{1}^{\prime}\right)+\sum_{k=1}^{\frac{n-7}{3}} \tau\left(K_{3 k-1}^{\prime}\right)+\sum_{k=1}^{\frac{n-7}{3}} \tau\left(K_{3 k}^{\prime}\right)+\tau\left(<V^{\prime \prime}>\right) \\
& =\frac{n-1}{3}+\frac{(n-7)(n+8)}{18}+\frac{(n-7)(n+5)}{9}+7 \\
& =\frac{n(n+1)}{6}-\frac{1}{3} \\
& =\left[\frac{n(n+1)}{6}\right]
\end{aligned}
$$

case (iii) $(n \equiv 2(\bmod 3))$. In this case we set $V^{\prime}=\bigcup_{i=1}^{n-5} V\left(K_{i}^{\prime}\right)$ and $V^{\prime \prime}=\bigcup_{i=n-4}^{n} V\left(K_{i}^{\prime}\right)$ so that $V^{\prime} \cup V^{\prime \prime}=V$ and $V^{\prime} \cap V^{\prime \prime}=\phi$. Now $V^{\prime}=\bigcup_{i=1}^{n-5} V\left(K_{i}^{\prime}\right)$ $=\bigcup_{k=1}^{\frac{n-5}{3}} V\left(K_{3 k-2}^{\prime}\right) \bigcup_{k=1}^{\frac{n-5}{3}} V\left(K_{3 k-1}^{\prime}\right) \bigcup_{k=1}^{\frac{n-5}{3}} V\left(K_{3 k}^{\prime}\right)$

(ie.) $\left.\tau\left(<V^{\prime}\right\rangle\right)=\sum_{k=1}^{\frac{n-5}{3}} \tau\left(K_{3 k-2}^{\prime}\right)+\sum_{k=1}^{\frac{n-5}{3}} \tau\left(K_{3 k-1}^{\prime}\right)+\sum_{k=1}^{\frac{n-5}{3}} \tau\left(K_{3 k}^{\prime}\right)$

For each $k\left(1 \leq k \leq \frac{n-5}{3}\right),\left|V\left(K_{3 k-2}^{\prime}\right)\right|=n-3 k+2+1=n-3 k+3 \equiv 2(\bmod 3)$ and $\left|V\left(K_{3 k-1}^{\prime}\right)\right|=n-3 k+1+1=n-3 k+2 \equiv 1(\bmod 3)$. Let $K_{3 k-2}^{\prime \prime}=$ $K_{3 k-2}^{\prime}-\left\{e_{(3 k-2)(3 k-1)}, e_{(3 k-2)(3 k)}\right\}$ and $K_{3 k-1}^{\prime \prime}=K_{3 k-1}^{\prime}-\left\{e_{(3 k-1)(3 k)}\right\}$. It is clear that $\left|V\left(K_{3 k-2}^{\prime \prime}\right)\right|=\left|V\left(K_{3 k-1}^{\prime \prime}\right)\right|=n-3 k+1 \equiv 0(\bmod 3)$ for each $k\left(1 \leq k \leq \frac{n-5}{3}\right)$. Also $<\left\{e_{(3 k-2)(3 k-1)}, e_{(3 k-2)(3 k)}, e_{(3 k-1)(3 k)}\right\}>$ is a $3-$ cycle. $\left|V\left(K_{3 k}^{\prime}\right)\right|=n-3 k+1 \equiv 0(\bmod 3)$ and hence $\tau\left(K_{3 k}^{\prime}\right)=\frac{1}{3}(n-3 k+1)$ for each $k\left(1 \leq k \leq \frac{n-5}{3}\right)$. Hence

$$
\begin{aligned}
\tau\left(<V^{\prime}>\right) & =\sum_{k=1}^{\frac{n-5}{3}} \tau\left(K_{3 k-2}^{\prime}\right)+\sum_{k=1}^{\frac{n-5}{3}} \tau\left(K_{3 k-1}^{\prime}\right)+\sum_{k=1}^{\frac{n-5}{3}} \tau\left(K_{3 k}^{\prime}\right) \\
& =\sum_{k=1}^{\frac{n-5}{3}}\left(\frac{1}{3}\left|V\left(K_{3 k-2}^{\prime \prime}\right)\right|+\frac{1}{3}\left|V\left(K_{3 k-1}^{\prime \prime}\right)\right|+1\right)+\sum_{k=1}^{\frac{n-5}{3}} \frac{1}{3}\left|V\left(K_{3 k}\right)\right| \\
& =\sum_{k=1}^{\frac{n-5}{3}}\left(\frac{1}{3}(n-3 k+1)+\frac{1}{3}(n-3 k+1)+1\right)+\sum_{k=1}^{\frac{n-5}{3}} \frac{1}{3}(n-3 k+1) \\
& =\frac{(n-5)(n+6)}{6}
\end{aligned}
$$


$V^{\prime \prime}=\bigcup_{i=n-4}^{n} V\left(K_{i}^{\prime}\right)$ and hence $\left|V^{\prime \prime}\right|=\sum_{i=n-4}^{n}(n-i+1)=15 \equiv 0(\bmod 3)$ and the following is the collection of disjoint $3-$ cycles of $\left\langle V^{\prime \prime}\right\rangle$.

$\mathbb{C}=\left\{v_{n-4} e_{(n-4)(n-3)} e_{(n-4) n}, v_{n-3} e_{(n-3)(n-2)} e_{(n-3) n}, v_{n-2} e_{(n-4)(n-2)} e_{(n-2)(n-1)}\right.$, $\left.v_{n-1} e_{(n-4)(n-1)} e_{(n-3)(n-1)}, v_{n} e_{(n-2) n} e_{(n-1) n}\right\}$.

Hence $\left\langle V^{\prime \prime}\right\rangle=5$ and

$$
\begin{aligned}
\tau\left(M\left(K_{n}\right)\right) & \geq \tau\left(<V^{\prime}>\right)+\tau\left(<V^{\prime \prime}>\right) \\
& =\frac{(n-5)(n+6)}{6}+5 \\
& =\frac{n(n+1)}{6} .
\end{aligned}
$$

Hence for all the cases $\tau\left(M\left(K_{n}\right)\right) \geq\left[\frac{n(n+1)}{6}\right]$. Since $\left|V\left(M\left(K_{n}\right)\right)\right|=\frac{n(n-1)}{2}+$ $n=\frac{n(n+1)}{2}, \tau\left(M\left(K_{n}\right)\right) \leq\left[\frac{n(n+1)}{6}\right]$. Therefore $\tau\left(M\left(K_{n}\right)\right)=\left[\frac{n(n+1)}{6}\right]$.

Since $V\left(T\left(K_{n}\right)\right)=V\left(M\left(K_{n}\right)\right)$ and $\tau\left(M\left(K_{n}\right)\right)=\frac{1}{3}\left|V\left(M\left(K_{n}\right)\right)\right|$ it can be concluded that $\tau\left(T\left(K_{n}\right)\right)=\tau\left(M\left(K_{n}\right)\right)$. Each vertex $v_{i}$ of a graph $G$ with degree $\operatorname{deg} v_{i}$ induce a clique of order $\operatorname{deg} v_{i}+1$ in $M(G)$. (ie.) At each vertex $v_{i}$ of $G$, we count $\left[\frac{\operatorname{deg} v_{i}+1}{3}\right]$ vertex disjoint cycles in $M(G)$.

This argument yields an upper bound for the tulgeity of middle graph of any graph.

Theorem 3.2. For any graph $G, \tau(M(G)) \leq \sum_{i}\left[\frac{\operatorname{deg} v_{i}+1}{3}\right]$

Theorem 3.3. If $T$ is a tree and for each pair of vertices $\left(v_{i}, v_{j}\right)$ with $\operatorname{deg} v_{i}, \operatorname{deg} v_{j}>2$ if there exists a vertex $v$ of degree 2 on $P\left(v_{i}, v_{j}\right)$ then $\tau(M(T))=\sum_{i}\left[\frac{\operatorname{deg} v_{i}+1}{3}\right]$

Proof. Let $T$ be a tree satisfying the given condition. Each vertex $v_{i}$ of $T$ of degree $>2$ induce a clique of order deg $v_{i}+1$ in $M(T)$. Since for each pair of vertices $\left(v_{i}, v_{j}\right)$ of $T$ with degree $>2$, there exists a vertex $v$ of degree 2 on $P\left(v_{i}, v_{j}\right)$, there exists a bridge joining the corresponding cliques in $M(T)$ and hence $\tau(M(T))=\sum_{i}\left[\frac{\operatorname{deg} v_{i}+1}{3}\right]$ 


\section{Tulgeity of Middle graph of $K_{m, n}$}

Theorem 4.1. $\tau\left(M\left(K_{m, n}\right)\right)=\left[\frac{m+n+m n}{3}\right]$ for any $m, n \geq 4$

Proof. Let $V\left(K_{m, n}\right)=\left\{u_{i}: 1 \leq i \leq m\right\} \cup\left\{v_{j}: 1 \leq j \leq n\right\}$ and $E\left(K_{m, n}\right)=\left\{e_{i j}=u_{i} v_{j}: 1 \leq i \leq m, 1 \leq j \leq n\right\}$. It is clear that $e_{i j} \neq e_{j i}$ for any $i \neq j$. By the definition of middle graph, $M\left(K_{m, n}\right)$ has the vertex set $V\left(M\left(K_{m, n}\right)\right)=V\left(K_{m, n}\right) \cup E\left(K_{m, n}\right)$.

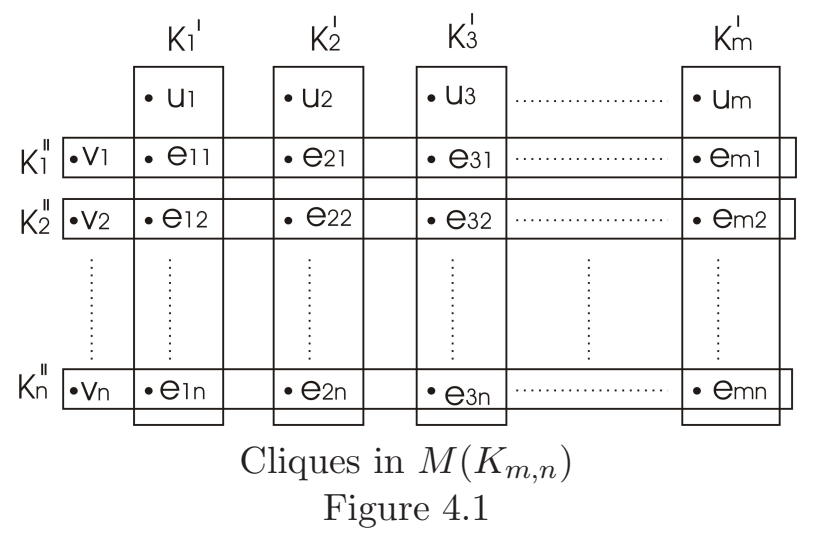

For each $i(1 \leq i \leq m), u_{i}$ is adjacent with all the vertices of $\left\{e_{i j}: 1 \leq j \leq n\right\}$ and for each $j(1 \leq j \leq n), v_{j}$ is adjacent with each vertex of $\left\{e_{i j}: 1 \leq j \leq m\right\}$. Also $e_{i j} e_{k l} \in E\left(M\left(K_{m, n}\right)\right)$ if and only if either $i=k$ or $j=l$.

For each $i$, the set of vertices $\left\{e_{i j}: 1 \leq j \leq n\right\} \cup\left\{u_{i}\right\}$ of $M\left(K_{m, n}\right)(1 \leq i \leq m)$ induces a clique of order $n+1$ ( say $k_{i}^{\prime}$ ). Similarly for each $j$, the set of vertices $\left\{e_{i j}: 1 \leq j \leq m\right\} \cup\left\{v_{j}\right\}$ of $M\left(K_{m, n}\right)(1 \leq j \leq n)$ induces a clique of order $m+1\left(\right.$ say $\left.k_{j}^{\prime} \prime\right)$. Also $<\left\{u_{i}: 1 \leq i \leq m\right\}$ and $<\left\{v_{j}: 1 \leq j \leq n\right\}>$ are totally disconnected in $M\left(K_{m, n}\right)$.

Vertex disjoint 3-cycles of $M\left(K_{m, n}\right)$ for the case of $4 \leq m, n \leq 5$ is shown in the figure. 


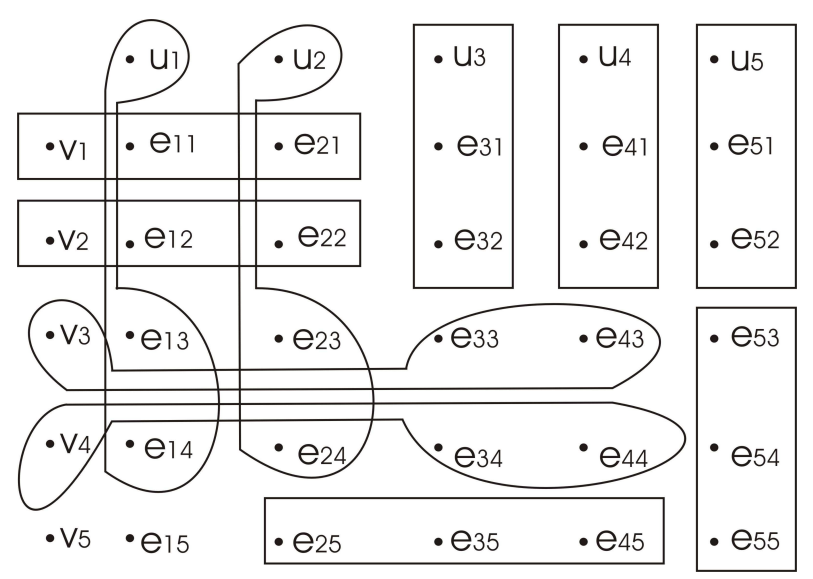

Disjoint 3-cycles in $M\left(K_{5,5}\right)$

Figure 4.2

Let $n \equiv k(\bmod 3)$ and $m \equiv l(\bmod 3)$ and $m, n \geq 6$. We choose the partition of $V\left(M\left(K_{m, n}\right)\right)$ such as $V=V^{\prime} \cup V^{\prime \prime} \cup V^{\prime \prime \prime}$ where

$$
\begin{aligned}
V^{\prime} & =\left\{v_{j}: 1 \leq j \leq n-2\right\} \bigcup_{i=1}^{2}\left\{u_{i}, e_{i j}: 1 \leq j \leq n\right\} \\
& =\left\{u_{1}, u_{2}\right\} \bigcup_{j=1}^{n}\left\{v_{j}, e_{i j}: 1 \leq i \leq 2\right\}-\left\{v_{n-1}, v_{n}\right\} \\
V^{\prime \prime} & =\bigcup_{i=3}^{m}\left\{u_{i}, e_{i j}: 1 \leq j \leq n-k-4\right\} \\
V^{\prime \prime \prime} & =\bigcup_{i=3}^{m}\left\{e_{i j}: n-k-3 \leq j \leq n\right\} \cup\left\{v_{n-1}, v_{n}\right\}
\end{aligned}
$$

Here $\tau\left(M\left(K_{m, n}\right)\right) \geq \tau\left(<V^{\prime}>\right)+\tau\left(<V^{\prime \prime}>\right)+\tau\left(<V^{\prime \prime \prime}>\right)$

Since for each $j(1 \leq j \leq n-2),<\left\{v_{j}, e_{i j}: 1 \leq i \leq 2\right\}>$ is a 3 -cycle, there exists $n-2$ disjoint $3-$ cycle in $\left\langle V^{\prime}\right\rangle$ and also $\left\langle V^{\prime}\right\rangle$ two $3-$ cycles $<\left\{u_{1}, e_{1(n-1)}, e_{1 n}\right\}>$ and $<\left\{u_{2}, e_{2(n-1)}, e_{2 n}\right\}>$. Hence $\tau\left(<V^{\prime}>\right)=n$. Since for each $i(3 \leq i \leq m),<\left\{u_{i}, e_{i j}: 1 \leq j \leq n-k-4\right\}>$ is a subgraph of the clique $K_{i}^{\prime}$, there exists $\frac{1}{3}(n-k-3)$ disjoint 3 -cycles in $<\left\{u_{i}, e_{i j}: 1 \leq j \leq n\right.$ $-k-4\}>$.

(ie) $\tau\left(<V^{\prime \prime}>\right)=\sum_{i=3}^{m} \frac{1}{3}(n-k-3)=\frac{1}{3}(m-2)(n-k-3)$. Now we find the 
collection of vertex disjoint $3-$ cycles of $<V^{\prime \prime \prime}>$ as follows $\mathbb{C}=\left\{<\left\{v_{n-1}, e_{3(n-1)}, e_{4(n-1)}\right\}>,<\left\{v_{n}, e_{(m-1) n}, e_{m n}\right\}>\right\}$ $\mathbb{C}^{\prime}=\left\{<\left\{e_{i(n-3)}, e_{i(n-2)}, e_{i(n-1)}\right\}>: 5 \leq i \leq m\right\}$ $\mathbb{C}^{\prime \prime}=\left\{<\left\{e_{(5+l+i) n}, e_{(5+l+i+1) n}, e_{(5+l+i+2) n}\right\}>: i=3 p, 0 \leq p \leq \frac{m-l-9}{3}\right\}$. It is clear that the cycles of $\mathbb{C}, \mathbb{C}^{\prime}$ and $\mathbb{C}^{\prime \prime}$ are vertex disjoint and $|\mathbb{C}|=2,\left|\mathbb{C}^{\prime}\right|=$ $m-4,\left|\mathbb{C}^{\prime \prime}\right|=\frac{m-l-9}{3}+1$. If $V(\mathbb{C})$ denotes the set of vertices belonging to the cycles of $\mathbb{C}$ then we find the disjoint cycles of $V^{\prime \prime \prime}-\left(V(\mathbb{C}) \cup V\left(\mathbb{C}^{\prime}\right) \cup V\left(\mathbb{C}^{\prime \prime}\right)\right)$ ( say $V^{i v}$ ) in the following cases.

Since $K_{m, n} \approx K_{n, m}$, it is suffices to consider only the following six cases to find $\tau\left(<V^{\prime \prime \prime}>\right)$.

Case (i) $(k=0, l=0)$

In this case $V^{i v}=\left\{e_{3(n-3)}, e_{3(n-2)}, e_{3 n}, e_{4(n-3)}, e_{4(n-2)}, e_{4 n}\right\}$ and hence there exists two disjoint 3 -cycles in $\left\langle V^{i v}>\right.$. Hence $\tau\left(V^{\prime \prime \prime}\right)=|\mathbb{C}|+\left|\mathbb{C}^{\prime}\right|+\left|\mathbb{C}^{\prime \prime}\right|+2=$ $\frac{4 m-6}{3}$.

Therefore $\tau\left(M\left(K_{m, n}\right)\right) \geq n+\frac{1}{3}(m-2)(n-3)+\frac{4 m-6}{3}=\left[\frac{m+n+m n}{3}\right]$.

Case(ii) $(k=0, l=1)$

In this case $V^{i v}=\bigcup_{i=1}^{4}\left\{e_{i(n-3)}, e_{i(n-2)}, e_{i n}\right\} \cup\left\{e_{5 n}\right\}$. Since $<\left\{e_{i(n-3)}, e_{i(n-2)}, e_{i n}\right\}>$ is a 3 -cycle for each $i(3 \leq i \leq 4), \tau\left(<V^{\prime \prime \prime}>\right)=2$. Hence $\tau\left(<V^{i v}>\right)=$ $\frac{4 m-7}{3}$.

Therefore $\tau\left(M\left(K_{m, n}\right)\right) \geq n+\frac{1}{3}(m-2)(n-3)+\frac{4 m-7}{3}=\left[\frac{m+n+m n}{3}\right]$.

Case(iii) $(k=0, l=2)$

In this case $V^{i v}=\bigcup_{i=3}^{4}\left\{e_{i(n-3)}, e_{i(n-2)}, e_{i n}\right\} \cup\left\{e_{5 n}, e_{6 n}\right\}$ and hence $<V^{i v}>$ has 2 -vertex disjoint 3 -cycles. Hence $\tau\left(<V^{\prime \prime \prime}>\right)=\frac{4 m-8}{3}$ and $\tau\left(<V^{\prime \prime}>\right)=$ $\frac{1}{3}(m-2)(n-3)$. Hence $\tau\left(M\left(K_{m, n}\right)\right) \geq n+\frac{1}{3}(m-2)(n-3)+\frac{4 m-8}{3}=$ $\left[\frac{m+n+m n}{3}\right]$.

Case(iv) $(k=1, l=1)$ 
In this case $V^{i v}=\left\{e_{i(n-4)}: 5 \leq i \leq m\right\} \bigcup_{i=3}^{4}\left\{e_{i(n-4)}, e_{i(n-3)}, e_{i(n-2)}\right\} \cup\left\{e_{3 n}, e_{4 n}, e_{5 n}\right\}$.

It is clear that $\tau\left(\left\langle V^{i v}\right\rangle\right)=\frac{m-4}{3}+2+1=\frac{m+5}{3}$ and $\left.\tau\left(<V^{\prime \prime \prime}\right\rangle\right)=$ $\frac{5 m-8}{3}$.

$\tau\left(<V^{\prime \prime}>\right)=\frac{1}{3}(m-2)(n-4)$ and hence $\tau\left(M\left(K_{m, n}\right)\right) \geq n+\frac{1}{3}(m-2)(n-4)+$ $\frac{5 m-8}{3}=\left[\frac{m+n+m n}{3}\right]$.

$\operatorname{Case}(\mathbf{v})(k=1, l=2)$

In this case $V^{i v}=\left\{e_{i(n-4)}: 4 \leq i \leq m\right\} \bigcup_{i=4}^{5}\left\{e_{i(n-3)}, e_{i(n-2)}, e_{i n}\right\} \cup\left\{e_{5 n}, e_{6 n}, e_{7 n}\right\}$. $\left.\left.\tau\left(<V^{i v}\right\rangle\right)=\frac{m-3}{3}+2+1=\frac{m+6}{3} \cdot \tau\left(<V^{\prime \prime \prime}\right\rangle\right)=\frac{5 m-8}{3}$ and $\left.\tau\left(<V^{\prime \prime}\right\rangle\right)=$ $\frac{1}{3}(m-2)(n-4)$.

Hence $\tau\left(M\left(K_{m, n}\right)\right) \geq n+\frac{1}{3}(m-2)(n-4)+\frac{5 m-8}{3}=\left[\frac{m+n+m n}{3}\right]$.

Case(vi) $(k=2, l=2)$

In this case $V^{i v}=\bigcup_{i=3}^{m}\left\{e_{i(n-5)}, e_{i(n-4)}\right\} \bigcup_{i=3}^{4}\left\{e_{i(n-3)}, e_{i(n-2), e_{i n}}\right\} \cup\left\{e_{5 n}, e_{6 n}\right\}$.

$<\left\{e_{i(n-5)}: 3 \leq i \leq m\right)>$ and $<\left\{e_{i(n-4)}: 3 \leq i \leq m\right)>$ are the cliques of order $m-2$ and hence there exists $\frac{2}{3}(m-2)$ disjoint $3-$ cycles and $\left\{e_{i(n-3)}, e_{i(n-2)}\right.$, $\left.e_{i n}\right\}(3 \leq i \leq 4)$ induces 2 more disjoint 3 -cycles. Hence

$$
\tau\left(<V^{i v}>\right)=\frac{2}{3}(m-2)+2
$$

and

$$
\begin{gathered}
\tau\left(<V^{\prime \prime \prime}>\right)=2 m-4, \\
\tau\left(<V^{\prime \prime}>\right)=\frac{1}{3}(n-2-3)(m-2)=\frac{1}{3}(m-2)(n-5) .
\end{gathered}
$$

Therefore

$$
\tau\left(M\left(K_{m, n}\right)\right) \geq n+\frac{1}{3}(m-2)(n-5)+2 m-4=\left[\frac{m+n+m n}{3}\right] .
$$




\section{References}

[1] Akbar Ali. M.M, S. Panayappan, Tulgeity of Line Graphs, Journal of Mathematics Research, Vol 2(2), 2010, 146-149.

[2] Akbar Ali. M.M, S. Panayappan and Vernold Vivin.J, Tulgeity of Line, Middle and Total Graph of Wheel Graph Families, International Journal of Mathematical Combinatorics, Vol 3, 2010, 97-106.

[3] Gary Chartrand, Dennis Geller, Stephen Hedetniemi, Graphs with forbidden subgraphs. Journal of Combinatorial Theory Ser. B, 10, 1971, 12-41.

[4] Gary Chartrand, Hudson V. Kronk, Curtiss E. Wall, The point arboricity of a graph, Israel Journal of Mathematics, 6(2), 1968, 168-175.

[5] Frank Harary, Graph Theory, 1969, New Delhi: Narosa Publishing home.

[6] Anton Kundrik, Dual point partition number of complementary graphs. Mathematica Slovaca, 40(4), 1990, 367-374. 
